\title{
Outcomes of Ivermectin in the treatment of COVID-19: a systematic review and meta-
}

\section{analysis}

Alex Castañeda-Sabogal ${ }^{1,2}$, Diego Chambergo-Michilot ${ }^{3,4}$; Carlos J. Toro-Huamanchumo ${ }^{5,6}$, Christian Silva-Rengifo ${ }^{2,9}$, José Gonzales-Zamora ${ }^{7}$, Joshuan J. Barboza, ${ }^{8,9}$

\section{Afiliations}

${ }^{1}$ Universidad Privada Antenor Orrego, Facultad de Medicina, Escuela de Posgrado, Trujillo,

Perú; ${ }^{2}$ Universidad Privada Antenor Orrego, Facultad de Medicina, Trujillo, Perú; ${ }^{3}$ Universidad Científica del Sur, Lima, Perú; ${ }^{4}$ Red Latinoamericana de Cardiología, Lima, Perú; ${ }^{5}$ Universidad San Ignacio de Loyola, Unidad para la Generación y Síntesis de Evidencias en Salud; ${ }^{6}$ Clínica Avendaño, Unidad de Investigación Multidisciplinaria, Lima, Perú; ${ }^{7}$ University of Miami, Miller School of Medicine, Miami, Florida, USA; ${ }^{8}$ Universidad Señor de Sipán, Escuela de Medicina, Chiclayo, Perú; ${ }^{9}$ Tau-Relaped Group, Trujillo, Perú.

\section{Correspondence to:}

*Joshuan J. Barboza

E-mail: jbarbozameca@ relaped.com

\section{Statement of financial support:}

This research received no specific grant from any funding agency in the public, commercial, or not-for-profit sectors.

Disclosure statement: Authors declare that they have no conflict of interest. 


\begin{abstract}
Background: To assess the outcomes of ivermectin in ambulatory and hospitalized patients with COVID-19.
\end{abstract}

Methods: Five databases and websites for preprints were searched until January 2021 for randomized controlled trials (RCTs) and retrospective cohorts assessing ivermectin versus control in ambulatory and hospitalized participants. The primary outcome was overall mortality. Secondary outcome was recovered patients. For meta-analysis, random-effects and inverse variance meta-analyses with logarithmic transformation were performed. ROBINS-I for cohort studies, and the Cochrane Risk of Bias 2.0 tool for trials were used. The strength of evidence was assessed using GRADE.

Results. After the selection, twelve studies (five retrospective cohort studies, six randomized clinical trials and one case series), were included. In total, 7412 participants were reported, the mean age was 47.5 (SD 9.5) years, and 4283 (58\%) were male. Ivermectin was not associated with reduced mortality (logRR: $0.89,95 \%$ CI 0.09 to $1.70, \mathrm{p}=0.04, \mathrm{I}^{2}=84.7 \%$ ), or reduced patient recovery $\left(\operatorname{logRR} 5.52,95 \% \mathrm{CI}-24.36\right.$ to $\left.35.4, \mathrm{p}=0.51, \mathrm{I}^{2}=92.6 \%\right)$. All studies had a high risk of bias, and showed a very low certainty of the evidence.

Conclusions: There insufficient certainty and quality of evidence to recommend the use of ivermectin to prevent or treat ambulatory or hospitalized patients with COVID-19.

Keywords: Ivermectin, Treatment, COVID-19, SARS-Cov-2 


\section{INTRODUCTION}

Since the first reported case of severe respiratory syndrome coronavirus 2 (SARS-CoV-2), in December 2019 in Wuhan, China; cases of coronavirus disease (COVID-19) have increased exponentially, with more than 95 million infected people worldwide until January 18, 2021 (https://coronavirus.jhu.edu/map.html). This high volume of COVID-19 cases has led to several problems including an overburdened health system, and a worrisome shortage of healthcare personnel. In this setting, finding an effective therapy against SARS-CoV-2 has become an urgent need (1)

The current treatment of COVID-19 has been limited to general supportive care, because studies evaluating the efficacy of treatment in patients with COVID-19 have had several limitations and no treatment has demonstrated strong evidence for widespread recommendation (2).

Ivermectin is a semisynthetic anthelmintic agent that selectively binds to glutamate-gated chloride ion channels found in nerve and muscle cells of invertebrates (3). However, an antiviral activity in RNA and DNA viruses has also been reported (4).

Caly, et al. conducted an in vitro study, in which they inoculated the SARS-CoV-2 virus in Vero/hSLAM cells, and found that Ivermectin at a dose of $4 \mu \mathrm{M}$ reduced the viral load after 48 hours. This finding encouraged the conduction of studies aimed to evaluate the clinical effectiveness of Ivermectin (5).

On the other hand, Virginia D. Schmith et al. developed a pharmacokinetic model, with transit absorption, first-order elimination and weight as covariates in the central volume of distribution and clearance. These authors used approved doses of $200 \mu \mathrm{g} / \mathrm{kg}$ (in $3 \mathrm{mg}$ 
increments); $120 \mathrm{mg} \mathrm{MD}$ and $60 \mathrm{mg}$ three times weekly (every 72 hours) and concluded that the inhibitory concentration $\left(\mathrm{IC}_{50}\right)$ of ivermectin was not expected to inhibit SARS-CoV-2 in lung tissue even simulating with 10 times the approved dose in humans. Similar findings were reported by Caly et al $(5,6)$.

We conducted a systematic review and meta-analyses to evaluate the clinical efficacy of ivermectin for the treatment of COVID-19. This will provide clinicians with an overview of the scientific evidence on a potential treatment option, which will help in the clinical management of COVID-19 patients. 


\section{METHODS}

\section{Protocol}

This systematic review was reported according to the Preferred Reporting Items for Systematic Reviews and Meta-Analyses (PRISMA) (7).

\section{Data sources}

We searched PubMed, Scopus, Web of Science, Ovid-Medline, Embase, websites for preprints/preproofs (“Other $\quad$ sources”; $\quad$ https://www.medrxiv.org, https://preprints.scielo.org/index.php/scielo, https://www.biorxiv.org, https://arxiv.org), websites for protocols of clinical trials (https://clinicaltrials.gov). We performed a search strategy for each database. The complete search strategy is found in Supplementary file. We included all the original published studies (either as preprints or in scientific journals) of clinical trials, non-randomized studies of intervention, and retrospective cohorts, without language restrictions, from inception to January 21, 2020; that have included patients (ambulatory or hospitalized) with COVID-19, and have compared a group that received ivermectin with a group that did not; regardless of their study design. Systematic reviews, narrative reviews, conference proceedings, editorials, and letters to the editor without original data were excluded.

\section{Outcomes}

Primary outcome was overall mortality. Secondary outcome was recovered patients.

\section{Study selection}

Two authors (JJB, DCM) independently screened search results by title and abstract according to the inclusion and exclusion criteria, using a web program Rayyan (rayyan- 
qcri.org). Also, two authors (JJB, DCM) independently assessed relevant studies and selected by full-text for the next phase of assessment. Discrepancies were consulted with another author (ACS), and a consensus was reached. The selection of articles in each stage of the review process was made using the Endnote X9 software.

\section{Data extraction}

Two authors (JJB, DCM) independently extracted the data using pre-piloted Excel spreadsheets. Again, discrepancies were consulted with another author (ACS). The data extracted from each study were: Author, year, country, type of study, number of patients with ivermectin treatment, treatment/comparison or control arm, characteristics and condition of the patient when the treatment was received, methods of assessment and confounding variables, outcomes, and absolute effect of ivermectin versus control. Regarding the outcome of recovered patients, this variable was evaluated according to the criteria considered by the authors of each included study.

\section{Risk of bias assessment}

Two investigators (JJB, DCM) independently assessed the risk of bias by using the ROBINS-I (Risk Of Bias In Non-Randomized Studies of Interventions) tool (8) for cohort studies and the Cochrane Risk of Bias 2.0 tool (9) for trials; disagreements were resolved by discussion with a third investigator (ACS).

\section{Data synthesis and statistical analysis}

We assessed the certainty of evidence using the GRADE methodology (10). When possible, we meta-analyzed results of RCTs and non-randomized studies that have used methods to control by possible confounders. 
Random-effects models with Hartung-Knapp adjustment for random effects model, and the inverse variance method were used for all meta-analyses. Effects of ivermectin were described with log relative risks (LogRRs) with 95\% confidence intervals (Log RR 95\% CIs) for dichotomous outcomes in the observational studies that were assessed. Heterogeneity among studies was assessed using the $\mathrm{I}^{2}$ statistic: 0-30\% meant low, 30$60 \%$ moderate, and $>60 \%$ high heterogeneity (11). Subgroup analysis by year $(<60$ years vs $>60$ years), condition (outpatient vs inpatient), and need of oxygen support (with vs without oxygen support) was proposed; however, due to the insufficient published evidence, these aspects were not evaluated. A sensitivity analysis was performed excluding those studies without adjustment for confounding. (12).

\section{Ethical considerations}

This is a systematic review of published and open information, in which no human subjects participated. No ethics committee approval was required. 


\section{Results}

\section{Selection of studies}

The search yielded 532 results. After duplicates were excluded, 232 titles and abstracts were reviewed, 210 of these were excluded, and 22 scientific papers were evaluated in detail.

Finally, 12 studies were included in the qualitative synthesis (13-24), and five studies were included in the quantitative synthesis (Figure 1). Most studies were pre-print studies and two studies $(18,22)$ were anticipated results of clinical trials protocols.

\section{Characteristics of the studies included}

Main characteristics of included studies are summarized in Table 1. Two studies were located in USA $(21,22)$, two from South America $(15,23)$, one from Iraq (16), two from Spain, one from Iran, and four from Bangladesh $(13,17,18,20)$. Five retrospective cohort studies (14, $16,17,21,23,25)$, six clinical trials $(13,18-20,22,24)$, and one case series (15) were found. There were 7412 reported participants, the mean age was 47.5 (SD 9.1) years, and 4283 $(58 \%)$ were male. The treatment was ivermectin (alone or with azithromycin, hydroxychloroquine, dexamethasone, enoxaparin, aspirin or dicloxacillin). Only one study reported no control group (15). Most patients were hospitalized and confirmed COVID-19 by RT-PCR, except for individuals included in one study evaluating asymptomatic families (22). Regarding methods of assessment and confounding variables, two studies analyzed the confounding variables by propensity score weighting and adjusted by age, sex, location, type of admission, comorbidities, antibiotics applied and other drugs $(21,23)$. One study assessed these variables by logistic and Cox regression adjusted by age, sex, comorbidities and use of other drugs (21). One study assessed the variables by Kaplan Meier survival curve, adjusted by age, gender, and severity (16). Finally, eight studies were not adjusted by confounding 
variables because they only applied bivariate analysis (13-15, 17-20, 22). Different outcomes were evaluated by included studies, and most studies have assessed mortality and recovered patients as the primary outcome. Only four studies did not describe data regarding mortality and recovery in their analysis $(14,15,22,26)$.

\section{Assessment of risk of bias}

Five RCT had high risk of bias due to missing outcome data (18-20, 22, 26). Four cohorts had serious risk of bias: Two studies were at serious risk of bias due to classification of interventions $(16,23)$, and two studies had critical risk of bias due to confounding $(14,17)$.

\section{Primary and secondary outcomes of ivermectin in patients with COVID-19}

In this analysis with four pre-print retrospective studies, and high risk of bias, ivermectin is not associated with reduced mortality $\left(\operatorname{logRR} 0.89,95 \%\right.$ CI 0.09 to $1.70, p=0.04, \mathrm{I}^{2}=84.7 \%$, Figure 2a). Additionally, ivermectin was not associated with reduced patient recovery $(\operatorname{logRR}$ $5.52,95 \%$ CI -24.36 to $35.4, p=0.51, \mathrm{I}^{2}=92.6 \%$, Figure $\left.2 \mathrm{~b}\right)$.

\section{Sensitivity analysis}

No differences were found between the overall analysis and that proposed in the sensitivity analysis in terms of outcomes.

\section{Certainty of evidence in included studies}

For certainty of evidence and evaluation of study quality, the GRADE recommendation was used. Two outcomes were assessed: Mortality (3607 participants, 5 retrospective studies), and recovery (397 participants, 3 pre-print retrospective studies). Both showed a very low certainty of the evidence, based on study design, risk of bias, inconsistency, indirectness and imprecision (Table 2). 


\section{Discussion}

\section{Main results}

We did not find a significantly reduction in the mortality and recovery of patients in the analyzed studies. It should be noted that the included studies are pre-print, so the information may vary and change the overall effect in our meta-analysis (although this trend may still be not significant).

Ivermectin has been widely used on the basis of having an antiviral effect against SARS-Cov2 (27). In this regard, a study published in Australia showed in-vitro effectiveness of ivermectin in Vero-cells; however, its clinical application in humans is very doubtful (5). This study was rapidly adopted by clinical practice guidelines, recommending ivermectin for the treatment of patients hospitalized with COVID-19, especially in countries severely affected by the pandemic. For example, Peru, one of the countries hardest hit by the pandemic, included Ivermectin as a first-line treatment, even as prophylaxis (28).

The rationale to include this drug was based on its pharmacologic properties and application in other scenarios. Ivermectin belongs to the chemical group of avermectins, and is widely used in large animals for the treatment and control of parasitic infections, and to assist on the treatment of scabies and ticks. In humans, ivermectin has been used as a prophylactic drug in filariasis and as a therapeutic agent for scabies. It is a drug approved by the FDA and has shown to be safe in the recommended dosages $(200 \mu \mathrm{g} / \mathrm{kg})(29)$.

Despite the published theoretical information, there are not enough clinical trials to confirm the efficacy and safety of ivermectin for prophylaxis or treatment of patients with COVID-19. A systematic review was performed by Padhy et al (30), and analyzed the effects of ivermectin in 629 patients with COVID-19 (4 observational studies were included). The ivermectin-treated group had 233 mild cases and 104 moderate to severe cases. All-cause 
mortality was reduced in 2 out of the 3 included studies (OR 0.53, 95\% CI 0.29-0.96). However, all these studies had a high risk of bias.

One of the limitations of the study conducted by Padhy et al. is that the overall effect of ivermectin on mortality was analyzed without considering the reported effect measure . Furthermore, the analysis was performed without transforming the individual effect (from OR to LogOR, for example), thus the OR reported was overestimated.

In another systematic review with networked meta-analysis, the effects of ivermectin on mortality were analyzed, and only two studies were included. The authors reported a very close statistical significance in terms of association of ivermectin with lower mortality (OR $0.15,95 \%$ CI 0.04 to $0.57, \mathrm{p}=0.005)$; however, they pointed out that these data had very low certainty of evidence (31).

Despite the small amount of highly biased evidence that has been published supporting the efficacy of ivermectin, the specific human dose has not been established. Bray et al. evaluated in vitro whether an ivermectin concentration of $0.1 \mathrm{uM}$ (instead of $5 \mathrm{uM}$ ) can inhibit SARS-Cov-2 (32). In clinical studies, the dose has ranged from $120 \mathrm{uM} / \mathrm{kg}$ to $200 \mathrm{uM} / \mathrm{kg}$ per dose in the intramuscular or oral form $(33,34)$. However, high doses for humans have not been approved (https://www.fda.gov/animal-veterinary/product-safety-information/faq-covid-19-andivermectin-intended-animals).

It is important to know that testing the efficacy of ivermectin in human clinical trials or observational study requires a previous evaluation in a dose-response trial, applying low dose (with less likelihood of pharmacological effect) and high dose relative to placebo. Given the lack of this type of studies, the ideal high dose of ivermectin has not been determined yet. 
Our study has some limitations that are worthy to mention. First, regarding heterogeneity, we found that five out of the eight studies were done in inpatients, two studies focused on outpatients only, and one study focused on both outpatients and inpatients. Similarly, we have found differences between treatment arms, for example, five studies reported the use of ivermectin by itself compared to the standard of care, and the remaining studies used ivermectin in combination with other drugs (dexamethasone, hydroxychloroquine, azithromycin). Although the outcome in hospitalized patients was overall mortality or recovery time, in outpatients the outcome was appearance of symptoms of Covid-19, except in one study (Carvallo) that measured disease severity and mortality. As observed, there is clinical heterogeneity that makes it difficult to combine the estimates in a pooled estimate.

It is possible that the methodological heterogeneity and biases found in this systematic review and meta-analysis provided results inconsistent with reality. For this reason, it was proposed to meta-analyze the outcomes separately, assuming for each of them the model of random effects, and thus having greater precision in the effect. In spite of having statistical heterogeneity, the analysis of biases performed on the selected studies and the measurement of the size of the effect according to the outcome made a very close approximation to the reality.

Finally, the LogRR and confidence intervals for mortality and recovery were found to be nonsignificant, and by applying GRADE, we determined the certainty of the evidence for this estimated effect: The true effect is likely to be substantially different from the estimated effect. This systematic review and meta-analysis concludes that more randomized clinical trials need to be included in a meta-analysis, with fewer biases to approximate more to the real measurable effect. At the moment, there is no evidence that the use of ivermectin changes the clinical outcome of inpatients or outpatients. 
medRxiv preprint doi: https://doi.org/10.1101/2021.01.26.21250420; this version posted January 27, 2021. The copyright holder for this preprint (which was not certified by peer review) is the author/funder, who has granted medRxiv a license to display the preprint in perpetuity.

It is made available under a CC-BY-NC-ND 4.0 International license.

\section{References}

1. Banerjee K, Nandy M, Dalai CK, Ahmed SN. The Battle against COVID 19 Pandemic: What we Need to Know Before we "Test Fire" Ivermectin. Drug Res (Stuttg). 2020;70(8):33740 .

2. Wiersinga WJ, Rhodes A, Cheng AC, Peacock SJ, Prescott HC. Pathophysiology, Transmission, Diagnosis, and Treatment of Coronavirus Disease 2019 (COVID-19): A Review. JAMA. 2020;324(8):782-93.

3. Dixit A, Yadav R, Singh AV. Ivermectin: Potential Role as Repurposed Drug for COVID19. Malays J Med Sci. 2020;27(4):154-8.

4. Heidary F, Gharebaghi R. Ivermectin: a systematic review from antiviral effects to COVID-19 complementary regimen. J Antibiot (Tokyo). 2020;73(9):593-602.

5. Caly L, Druce JD, Catton MG, Jans DA, Wagstaff KM. The FDA-approved drug ivermectin inhibits the replication of SARS-CoV-2 in vitro. Antiviral Res. 2020;178:104787.

6. Schmith VD, Zhou JJ, Lohmer LRL. The Approved Dose of Ivermectin Alone is not the Ideal Dose for the Treatment of COVID-19. Clin Pharmacol Ther. 2020;108(4):762-5.

7. Page M, McKenzie J, Bossuyt P, Boutron I, Hoffmann T, Mulrow C, et al. The PRISMA 2020 statement: an updated guideline for reporting systematic reviews. MetaArXiv Preprints. 2020.

8. Sterne JA, Hernán MA, Reeves BC, Savović J, Berkman ND, Viswanathan M, et al. ROBINS-I: a tool for assessing risk of bias in non-randomised studies of interventions. BMJ (Clinical research ed). 2016;355:i4919.

9. Sterne JAC, Savović J, Page MJ, Elbers RG, Blencowe NS, Boutron I, et al. RoB 2: a revised tool for assessing risk of bias in randomised trials. BMJ. 2019;366:14898.

10. Schünemann H BJ, Guyatt G, Oxman A, editors. GRADE handbook for grading quality of evidence and strength of recommendations. Updated October 2013. The GRADE Working Group, 2013. Group GW, editor. USA: GRADE Working Group; 2013.

11. Higgins JPT, Thompson SG, Deeks JJ, Altman DG. Measuring inconsistency in metaanalyses. BMJ (Clinical research ed). 2003;327(7414):557-60.

12. Higgins JPT TJ, Chandler J, Cumpston M, Li T, Page MJ, Welch VA. Cochrane Handbook for Systematic Reviews of Interventions. Chapter 24: Including non-randomized studies on intervention effects. Cochrane. 2020;Available from www.training.cochrane.org/handbook.

13. Ahmed S, Karim MM, Ross AG, Hossain MS, Clemens JD, Sumiya MK, et al. A five-day course of ivermectin for the treatment of COVID-19 may reduce the duration of illness. Int J Infect Dis. 2020;103:214-6.

14. Camprubi D, Almuedo-Riera A, Marti-Soler HI, Soriano A, Hurtado JC, Subira C, et al. Lack of efficacy of standard doses of ivermectin in severe COVID-19 patients. PLoS ONE. 2020;15(11 November):e0242184.

15. Carvallo HE, Hirsch RR, Farinella ME. Safety and Efficacy of the combined use of ivermectin, dexamethasone, enoxaparin and aspirin against COVID-19. medRxiv. 2020:2020.09.10.20191619.

16. Gorial FI, Mashhadani S, Sayaly HM, Dakhil BD, AlMashhadani MM, Aljabory AM, et al. Effectiveness of Ivermectin as add-on Therapy in COVID-19 Management (Pilot Trial). medRxiv. 2020:2020.07.07.20145979. 
17. Khan SI, Khan SI, Debnath CR, Nath PN, Mahtab MA, Nabeka H, et al. Ivermectin Treatment May Improve the Prognosis of Patients With COVID-19. Archivos de Bronconeumología (English Edition). 2020.

18. Mahmud R. A Phase III Trial to Promote Recovery From Covid 19 With Combined Doxycycline and Ivermectin Along Standard Care. Clinical trial registration. U.S. National Library of Medicine. Clinical Trials; 2020 2020/10/07/. Report No.: results/NCT04523831.

19. Morteza Shakhsi Niaee NG, et al. Ivermectin as an adjunct treatment for hospitalized adult COVID-19 patients: A randomized multi-center clinical trial. 2020.

20. Podder CH NC, Mohim Ibne Sina, Wasim Md Mohosin Ul Haque. Outcome of ivermectin treated mild to moderate COVID-19 cases: a single-centre, open-label, randomised controlled study. IMC Journal of Medical Science. 2020;14(2).

21. Rajter JC, Sherman MS, Fatteh N, Vogel F, Sacks J, Rajter JJ. Use of Ivermectin Is Associated With Lower Mortality in Hospitalized Patients With Coronavirus Disease 2019: The ICON Study. Chest. 2020.

22. Waheed S. Prophylactic Ivermectin in COVID-19 Contacts - Study Results ClinicalTrials.gov. US National Library of Medicine Clinical Trials. 2020.

23. Soto-Becerra P, Culquichicon C, Hurtado-Roca Y, Araujo-Castillo RV. Real-world effectiveness of hydroxychloroquine, azithromycin, and ivermectin among hospitalized COVID19 patients: results of a target trial emulation using observational data from a nationwide healthcare system in Peru. medRxiv. 2020:2020.10.06.20208066.

24. Chaccour C, Casellas A, Blanco-Di Matteo A, Pineda I, Fernandez-Montero A, RuizCastillo P, et al. The effect of early treatment with ivermectin on viral load, symptoms and humoral response in patients with non-severe COVID-19: A pilot, double-blind, placebocontrolled, randomized clinical trial. EClinicalMedicine. 2021:100720.

25. Cepelowicz J, Sherman M, Fatteh N, Vogel F, Sacks J, Rajter J-J. ICON (Ivermectin in COvid Nineteen) study: Use of Ivermectin is Associated with Lower Mortality in Hospitalized Patients with COVID19. medRxiv. 2020:2020.06.06.20124461.

26. Nosocomial infections in neonatal units in Turkey: Epidemiology, problems, unit policies and opinions of healthcare workers. Turkish Journal of Pediatrics. 2010;52(1):50-7.

27. Formiga FR, Leblanc R, de Souza Reboucas J, Farias LP, de Oliveira RN, Pena L.

Ivermectin: an award-winning drug with expected antiviral activity against COVID-19. J Control Release. 2020.

28. Chirinos JA, Corrales-Medina VF, Heresi-Dávila G, Hernandez AV, Málaga G, Mallea JM, et al. Sobre las recomendaciones del Ministerio de Salud para el tratamiento farmacológico de la COVID-19 en el Perú. Acta Médica Peruana. 2020;37:231-5.

29. Marcolino VA, Pimentel TC, Barao CE. What to expect from different drugs used in the treatment of COVID-19: A study on applications and in vivo and in vitro results. Eur J Pharmacol. 2020;887:173467.

30. Padhy BM, Mohanty RR, Das S, Meher BR. Therapeutic potential of ivermectin as add on treatment in COVID 19: A systematic review and meta-analysis. J Pharm Pharm Sci. 2020;23:462-9.

31. Kim MS, An MH, Kim WJ, Hwang TH. Comparative efficacy and safety of pharmacological interventions for the treatment of COVID-19: A systematic review and network meta-analysis. PLoS Med. 2020;17(12):e1003501.

32. Bray M, Rayner C, Noel F, Jans D, Wagstaff K. Ivermectin and COVID-19: A report in Antiviral Research, widespread interest, an FDA warning, two letters to the editor and the authors' responses. Antiviral Res. 2020;178:104805. 
medRxiv preprint doi: https://doi.org/10.1101/2021.01.26.21250420; this version posted January 27, 2021. The copyright holder for this preprint (which was not certified by peer review) is the author/funder, who has granted medRxiv a license to display the preprint in perpetuity. It is made available under a CC-BY-NC-ND 4.0 International license.

33. Gupta D, Sahoo AK, Singh A. Ivermectin: potential candidate for the treatment of Covid 19. Braz J Infect Dis. 2020;24(4):369-71.

34. Hosseini FS, Malektojari A, Ghazizadeh S, Hassaniazad M, Davoodian P, Dadvand H, et al. The efficacy and safety of Ivermectin in patients with mild and moderate COVID-19: A structured summary of a study protocol for a randomized controlled trial. Trials. 2021;22(1):4.

\section{Figure legends}

\section{Figure 1. PRISMA flowchart for included studies}

Figure 2. Forest plot for primary and secondary outcomes (a: Mortality; b: Recovered) 
Table 1. Characteristics of studies included

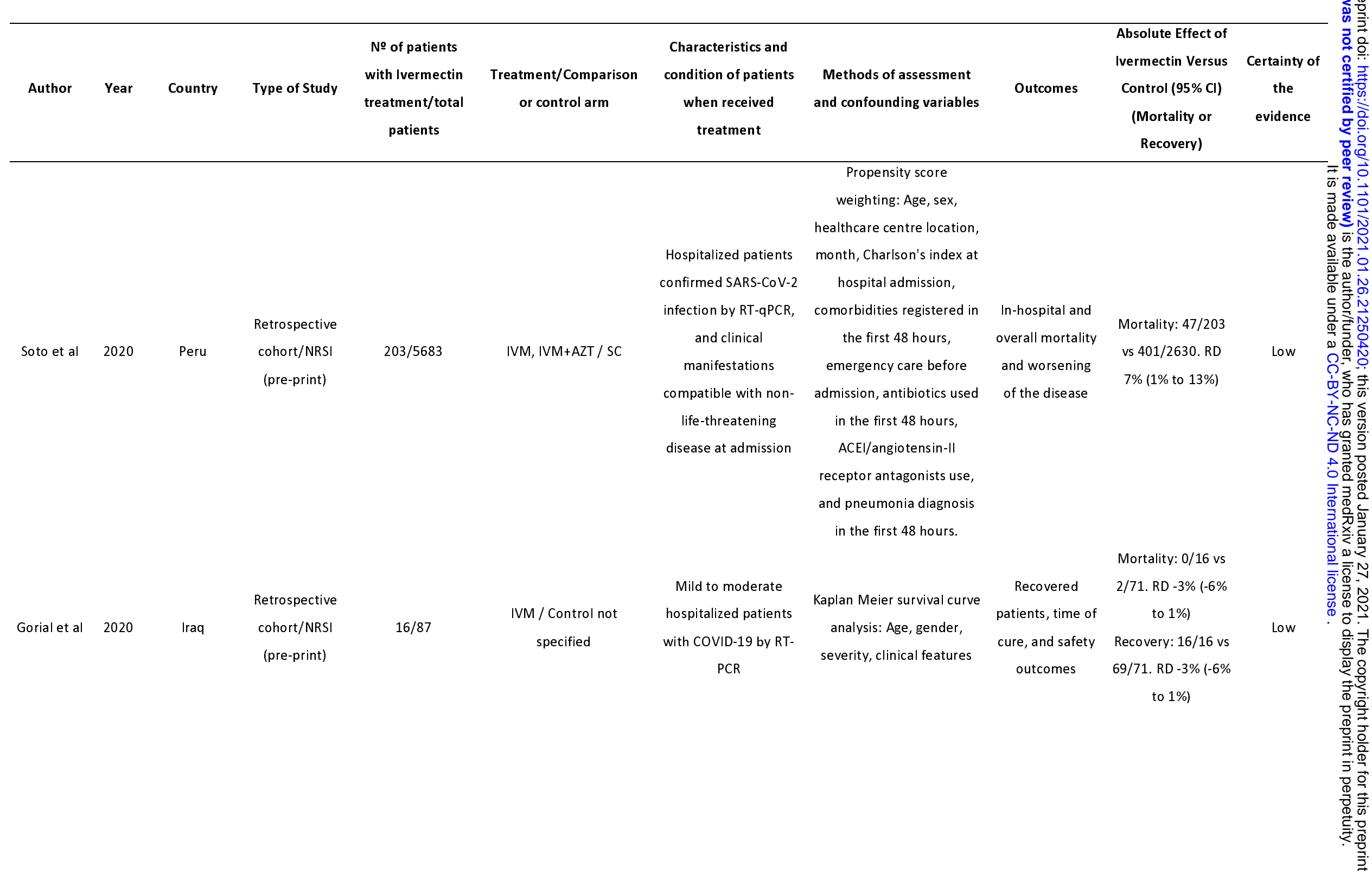




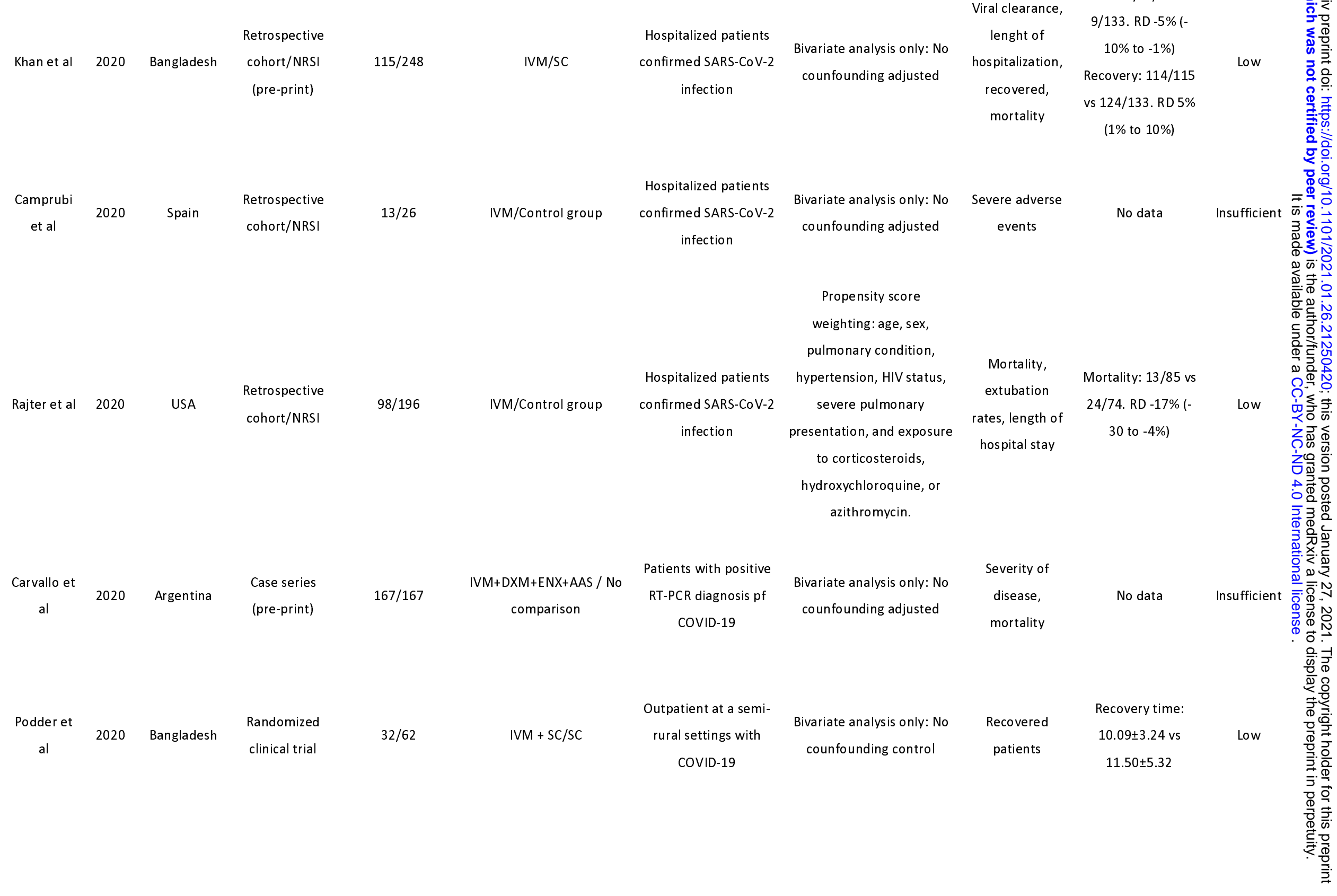




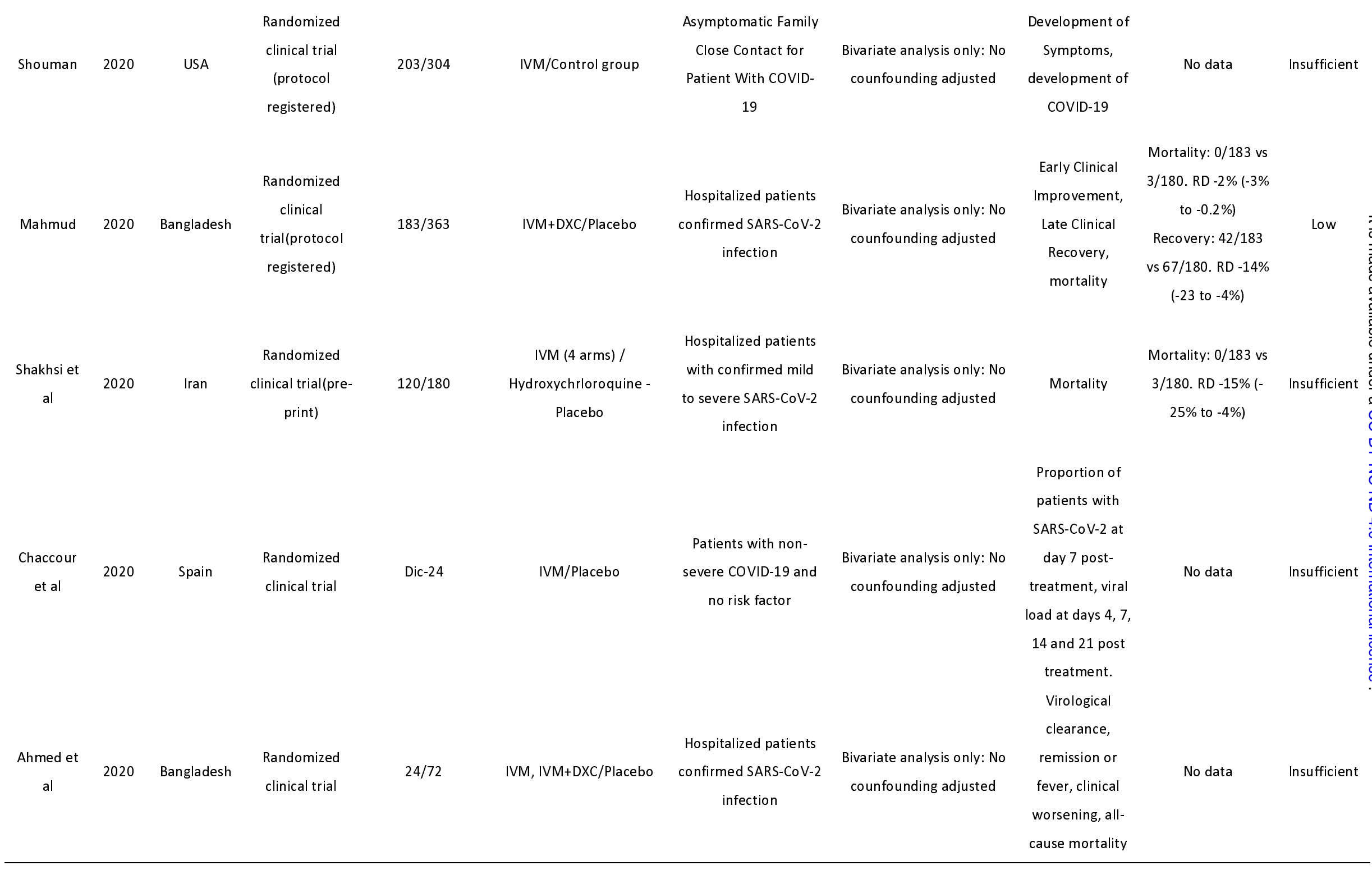




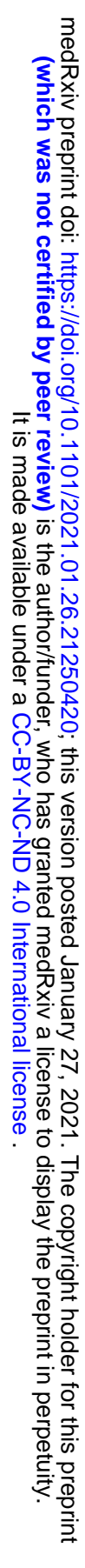


Table 2. Summary of certainty evidence

\begin{tabular}{|c|c|c|c|c|c|}
\hline \multirow[b]{2}{*}{ Outcomes } & \multirow{2}{*}{$\begin{array}{c}\text { № of participants } \\
\text { (studies) } \\
\text { Follow up }\end{array}$} & \multirow{2}{*}{$\begin{array}{c}\text { Certainty of the } \\
\text { evidence } \\
\text { (GRADE) }\end{array}$} & \multirow{2}{*}{$\begin{array}{l}\text { Relative effect } \\
\quad(95 \% \text { CI })\end{array}$} & \multicolumn{2}{|c|}{ Anticipated absolute effects } \\
\hline & & & & $\begin{array}{c}\text { Risk with [Standar } \\
\text { care] }\end{array}$ & $\begin{array}{l}\text { Risk difference with } \\
\text { [Ivermectin ] }\end{array}$ \\
\hline $\begin{array}{c}\text { Mortality } \\
\text { assessed with: RR }\end{array}$ & $\begin{array}{c}3607 \\
\text { (5 observational } \\
\text { studies) }\end{array}$ & $\begin{array}{c}\text { 丹19 } \\
\text { VERY LOW }\end{array}$ & $\begin{array}{c}\text { RR 0.70 } \\
(0.31 \text { to } 2.28)\end{array}$ & 154 per 1,000 & $\begin{array}{c}\mathbf{4 6} \text { fewer per } \mathbf{1 , 0 0 0} \\
\text { (106 fewer to } 197 \\
\text { more) }\end{array}$ \\
\hline $\begin{array}{c}\text { Recovery } \\
\text { assessed with: RR }\end{array}$ & $\begin{array}{c}397 \\
\left(\begin{array}{c}3 \text { observational } \\
\text { studies })\end{array}\right.\end{array}$ & $\begin{array}{c}\oplus \text { ? ? ? } \\
\text { VERY LOW }\end{array}$ & $\begin{array}{c}\text { RR } 1.37 \\
(0.61 \text { to } 3.07)\end{array}$ & 857 per 1,000 & $\begin{array}{c}317 \text { more per } \mathbf{1 , 0 0 0} \\
\text { (334 fewer to } 1,773 \\
\text { more })\end{array}$ \\
\hline
\end{tabular}

*The risk in the intervention group (and its $95 \%$ confidence interval) is based on the assumed risk in the comparison group and the relative effect of the intervention (and its $95 \% \mathrm{CI}$ ).

CI: Confidence interval; RR: Risk ratio

\section{GRADE Working Group grades of evidence}

High certainty: We are very confident that the true effect lies close to that of the estimate of the effect

Moderate certainty: We are moderately confident in the effect estimate: The true effect is likely to be close to the estimate of the effect, but there is a possibility that it is substantially different

Low certainty: Our confidence in the effect estimate is limited: The true effect may be substantially different from the estimate of the effect Very low certainty: We have very little confidence in the effect estimate: The true effect is likely to be substantially different from the estimate of effect 
Records identified through database searching $(n=532)$
Additional records identified through other sources

$$
(n=0)
$$

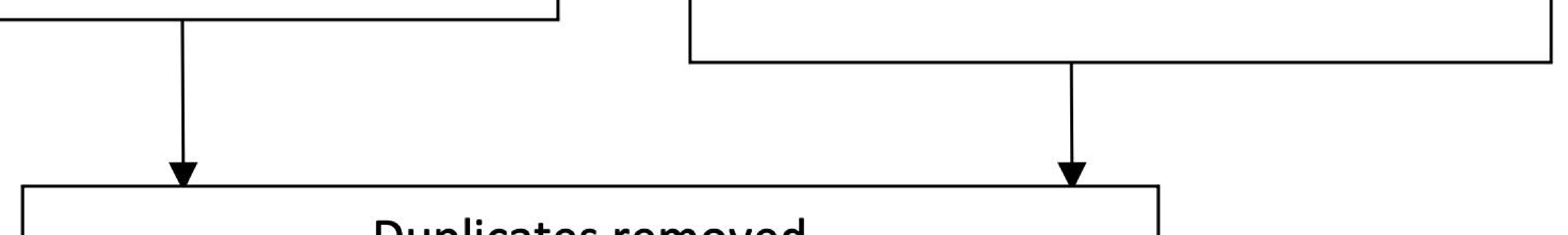

$$
(n=330)
$$

Studies included in qualitative synthesis

$$
(n=12)
$$

Full-text articles excluded

$$
(n=10)
$$

Letters: 9

Wrong outcomes: 1

Studies included in quantitative synthesis $(n=5)$ 
Study

Soto 2020

Cepelowicz 2020

Gorial 2020

Khan 2020

Rajter 2020

Random effects model

Heterogeneity: $I^{2}=85 \%, \tau^{2}=0.5290, p<0.01$
Log Risk Ratio

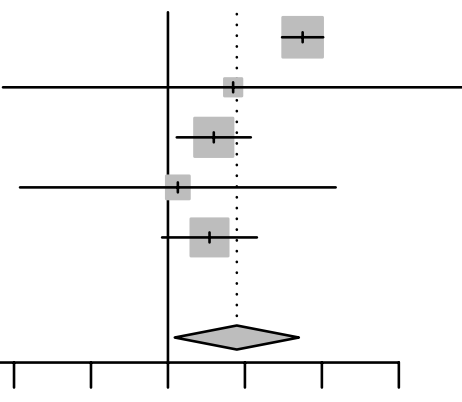

$\begin{array}{lllllll}-3 & -2 & -1 & 0 & 1 & 2 & 3\end{array}$
$\operatorname{logRR} \quad 95 \%-\mathrm{Cl}$ Weight

$1.75[1.48 ; 2.02] \quad 30.0 \%$ $0.85[-2.14 ; 3.84] \quad 5.8 \%$ $0.60[0.11 ; 1.08] 27.9 \%$ $0.13[-1.92 ; 2.18] \quad 10.1 \%$ $0.54[-0.08 ; 1.16] \quad 26.2 \%$

$0.89[0.09 ; 1.70] 100.0 \%$ 


\section{Study}

Cepelowicz 2020

Gorial 2020

Khan 2020

Random effects model

Heterogeneity: $I^{2}=93 \%, \tau^{2}=16.3960, p<0.01$
TE seTE

2.350 .5106

27.955 .3476

0.131 .0464
Log Risk Ratio

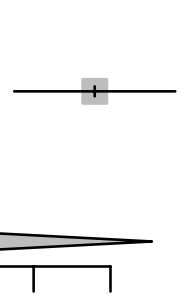

\section{$\log R R \quad 95 \%-C l$ Weight}

$2.35 \quad[1.35 ; 3.35] \quad 43.1 \%$

$27.95[17.47 ; 38.43] \quad 15.9 \%$

$0.13[-1.92 ; 2.18] \quad 41.0 \%$

$5.52[-24.36 ; 35.40] 100.0 \%$ 